\title{
ON THE STRUCTURE OF THE SET OF SOLUTIONS OF THE DARBOUX PROBLEM FOR HYPERBOLIC EQUATIONS
}

\author{
by F. S. DE BLASI and J. MYJAK \\ (Received 22nd October 1984)
}

\section{Introduction and main result}

Consider the Darboux problem

$$
\begin{aligned}
z_{x y} & =f(x, y, z) \\
z(x, 0) & =\phi(x), \quad z(0, y)=\psi(y),
\end{aligned}
$$

where $\phi, \psi: I \rightarrow R^{d}(I=[0,1])$ are given absolutely continuous functions with $\phi(0)=\psi(0)$, and the mapping $f: Q \times R^{d} \rightarrow R^{d}(Q=I \times I)$ satisfies the following hypotheses:

$\left(\mathrm{A}_{1}\right) f(., ., z)$ is measurable for every $z \in R^{d}$;

$\left(\mathrm{A}_{2}\right) f(x, y,$.$) is continuous for a.a. (almost all) (x, y) \in Q$;

$\left(\mathrm{A}_{3}\right)$ there exists an integrable function $\alpha: Q \rightarrow[0,+\infty)$ such that $|f(x, y, z)| \leqq \alpha(x, y)$ for every $(x, y, z) \in Q \times R^{d}$.

Let $C\left(Q, R^{d}\right)$ denote the Banach space of all continuous functions from $Q$ to $R^{d}$ endowed with the metric of uniform convergence.

By a solution of problem (1) we mean a function $z \in C\left(Q, R^{d}\right)$ satisfying

$$
z(x, y)=\phi(x)+\psi(y)-\phi(0)+\int_{0}^{x} \int_{0}^{y} f(\xi, \eta, z(\xi, \eta)) d \xi d \eta
$$

for every $(x, y) \in Q$.

The purpose of this note is to prove the following

Theorem. Let $f: Q \times R^{d} \rightarrow R^{d}$ satisfy $\left(\mathrm{A}_{1}\right),\left(\mathrm{A}_{2}\right),\left(\mathrm{A}_{3}\right)$. Let $\phi, \psi: I \rightarrow R^{d}$ be absolutely continuous functions with $\phi(0)=\psi(0)$. Then the set $\zeta_{\text {f }}$ of all solutions of the problem (1) is an $R_{\boldsymbol{\delta}}$-set in $C\left(Q, R^{d}\right)$.

Recall that a subset of a metric space is called an $R_{\delta}$-set if it is the intersection of a decreasing sequence of compact absolute retracts. It is known that an $R_{\delta}$-set is acyclic, in particular it is nonempty compact and connected.

Hukuhara [5] and Aronszajn [1] have proved that the set of solutions of the Cauchy problem $x^{\prime}=f(t, x), x(0)=x_{0}$, where $f: I \times R^{d} \rightarrow R^{d}$ is continuous and bounded, is an $R_{\delta^{-}}$ set in $C\left(I, R^{d}\right)$. Recently, by using topological degree arguments, Górniewicz and 
Pruszko [4] have established an analogous result for the Darboux problem (1), under the main hypothesis that $f$ be continuous with respect to all variables. In this note the set of solutions of problem (1) is shown to be an $R_{\delta}$-set also when $f$ satisfies hypotheses of Carathéodory type. Our approach is different from that used in [4].

Remark 1. The statement of the theorem remains true when the condition $\left(A_{3}\right)$ is replaced by the following one: there exist integrable functions $\alpha, \beta: Q \rightarrow[0,+\infty)$ such that $|f(x, y, z)| \leqq \alpha(x, y)+\beta(x, y)|z|$ for each $(x, y, z) \in Q \times R^{d}$.

\section{Preliminaries}

The following lemma can be proved as in [2, Lemma 2].

Lemma 1. Let $f: Q \times R^{d} \rightarrow R^{d}$ satisfy $\left(\mathrm{A}_{1}\right),\left(\mathrm{A}_{2}\right),\left(\mathrm{A}_{3}\right)$. Then for every $\varepsilon>0$ there exists a locally lipschitzian function $g: Q \times R^{d} \rightarrow R^{d}$ such that

$$
\iint_{Q} \sup _{z \in R^{d}}|g(\xi, \eta, z)-f(\xi, \eta, z)| d \xi d \eta<\varepsilon
$$

Recall that a subset $A$ of a metric space is called contractible if there exist a point $x_{0} \in A$ and a continuous function $h: I \times A \rightarrow A$ such that $h(0, x)=x_{0}$ and $h(1, x)=x$ for each $x \in A$.

Lemma 2 [6]. Let $A$ be a nonempty compact subset of a metric space $X$. Then $A$ is an $R_{\mathfrak{d}}$-set in $X$ if and only if $A$ is the intersection of a decreasing sequence of compact contractible subsets of $X$.

Let $L_{1}\left(Q, R^{d}\right)$ be the Banach space of the (equivalence classes of) Lebesgue integrable functions $v: Q \rightarrow R^{d}$, with the norm $\iint_{Q}|v(\xi, \eta)| d \xi d \eta$.

Lemma 3. Suppose that a sequence $\left\{v_{n}\right\} \subset L_{1}\left(Q, R^{d}\right)$ satisfies:

(i) $\left|v_{n}(x, y)\right| \leqq \alpha(x, y)$ for almost all $(x, y) \in Q\left(\alpha \in L_{1}\left(Q, R^{d}\right)\right)$;

(ii) for each $(x, y) \in Q$ the sequence

$$
\left\{\int_{0}^{x} \int_{0}^{y} v_{n}(\xi, \eta) d \xi d \eta\right\}
$$

is Cauchy.

Then $\left\{v_{n}\right\}$ is weakly Cauchy in $L_{1}\left(Q, R^{d}\right)$.

Proof. Clearly $\left\{v_{n}\right\}$ is norm bounded in $L_{1}\left(Q, R^{d}\right)$. Let $E$ be a measurable subset of $Q$. Let $\varepsilon>0$. Let $P \subset Q$ be an elementary set (that is a set which can be expressed as a union of a finite number of pairwise disjoint rectangles) such that $\iint_{E \Delta P} \alpha(\xi, \eta) d \xi d \eta<\varepsilon / 4$ $(E \Delta P=(E \backslash P) \cup(P \backslash E))$. As $P$ is an elementary set, by virtue of (ii) one can find an $n_{0} \in \mathbb{N}$ such that $\left|\iint_{P}\left(v_{m}(\xi, \eta)-v_{n}(\xi, \eta)\right) d \xi d \eta\right|<\varepsilon / 4$ if $m, n \geqq n_{0}$. Then, by an easy computation, 
one obtains

$$
\begin{aligned}
\mid \iint_{E}\left(v_{m}(\xi, \eta)\right. & \left.-v_{n}(\xi, \eta)\right) d \xi d \eta \mid \leqq 2 \iint_{E \Delta P} \alpha(\xi, \eta) d \xi d \eta \\
& +\left|\iint_{P}\left(v_{m}(\xi, \eta)-v_{n}(\xi, \eta)\right) d \xi d \eta\right|<2 \frac{\varepsilon}{4}+\frac{\varepsilon}{2}=\varepsilon
\end{aligned}
$$

$\left(m, n \geqq n_{0}\right)$, which shows that the sequence $\left\{\iint_{E} v_{n}(\xi, \eta) d \xi d \eta\right\}$ is Cauchy. By using [3, Theorem IV.8.7] one can complete the proof.

Denote by $\mathscr{K}$ the family of all nonempty compact convex subsets of $R^{d}$. Recall that a multifunction $G: Q \rightarrow \mathscr{K}$ is said to be measurable if the set $\{(x, y) \in Q \mid G(x, y) \cap U \neq \varnothing\}$ is (Lebesgue) measurable for every open subset $U$ of $R^{d}$. A multifunction $G: R^{d} \rightarrow \mathscr{K}$ is said to be upper semi-continuous (u.s.c.) if the set $\left\{u \in R^{d} \mid G(u) \subset U\right\}$ is open for every open subset $U$ of $R^{d}$.

Consider the (multivalued) Darboux problem

$$
\begin{aligned}
z_{x y} & \in F(x, y, z) \\
z(x, 0) & =\phi(x), \quad z(0, y)=\psi(y),
\end{aligned}
$$

where the functions $\phi, \psi: I \rightarrow R^{d}$ are as above, and the multifunction $F: Q \times R^{d} \rightarrow \mathscr{K}$ satisfies the following hypotheses:

$\left(\mathrm{H}_{1}\right) F(., ., z)$ is measurable for every $z \in R^{d}$;

$\left(\mathrm{H}_{2}\right) F(x, y,$.$) is u.s.c. for a.a. (x, y) \in Q$;

$\left(\mathrm{H}_{3}\right)$ there exists an integrable function $\alpha: Q \rightarrow[0,+\infty)$ such that $\sup \{|u| \mid u \in F(x, y, z)\} \leqq \alpha(x, y)$ for every $(x, y, z) \in Q \times R^{d}$.

By a solution of (3) we mean a function $z \in C\left(Q, R^{d}\right)$ such that there exists an integrable function $v: Q \rightarrow R^{d}$ satisfying

$$
v(x, y) \in F(x, y, z(x, y)) \text { for a.a. }(x, y) \in Q
$$

and

$$
z(x, y)=\phi(x)+\psi(y)-\phi(0)+\int_{0}^{x} \int_{0}^{y} v(\xi, \eta) d \xi d \eta \quad \text { for every } \quad(x, y) \in Q
$$

We denote by $\mu$ the Lebesgue measure in $R^{2}$ and by $B$ the unit closed ball in $R^{d}$.

Lemma 4. Let $F: Q \times R^{d} \rightarrow \mathscr{K}$ satisfy $\left(\mathrm{H}_{1}\right),\left(\mathrm{H}_{2}\right),\left(\mathrm{H}_{3}\right)$ and let $\phi, \psi: I \rightarrow R^{d}$ be absolutely continuous functions with $\phi(0)=\psi(0)$. In addition, suppose that there exists a locally lipschitzian function $g: Q \times R^{d} \rightarrow R^{d}$ such that $g(x, y, z) \in F(x, y, z)$ for each $(x, y, z) \in\left(Q \backslash Q_{0}\right) \times R^{d}$, where $\mu\left(Q_{0}\right)=0$. Then the set $\zeta_{F}$ of all solutions of problem (3) is $a$ (nonempty) compact contractible subset of $C\left(Q, R^{d}\right)$. 
Proof. Since the solution of problem (3) (with $g$ in the place of $f$ ) belongs to $\zeta_{F}$, one has that $\zeta_{F} \neq \varnothing$.

Let us show that $\zeta_{F}$ is compact. To this end consider any sequence $\left\{z_{n}\right\} \subset \zeta_{F}$. Taking into account the uniform continuity of $\phi, \psi$ and assumption $\left(\mathrm{H}_{3}\right)$ one can easily show that the functions $z_{n}$ are equicontinuous and equibounded. By Ascoli-Arzelà's Theorem, passing to a subsequence (without change of notation), we can assume that $\left\{z_{n}\right\}$ converges uniformly on $Q$, to a function $z_{0} \in C\left(Q, R^{d}\right)$. For each $n \in \mathbb{N}$ we have

$$
z_{n}(x, y)=\phi(x)+\psi(y)-\phi(0)+\int_{0}^{x} \int_{0}^{y} v_{n}(\xi, \eta) d \xi d \eta
$$

where $v_{n}(\xi, \eta) \in F\left(\xi, \eta, z_{n}(\xi, \eta)\right)$ for a.a. $(\xi, \eta) \in Q$. From (4) it follows that the sequence (2) converges for each $(x, y) \in Q$. Since $L_{1}\left(Q, R^{d}\right)$ is weakly complete, by Lemma 3 there exists a $v_{0} \in L_{1}\left(Q, R^{d}\right)$ such that $\left\{v_{n}\right\}$ converges weakly to $v_{0}$. By Mazur's Theorem there exists a sequence $\left\{w_{n}\right\}$ of finite convex combinations of $v_{n}$ 's

$$
w_{n}=\sum_{i=0}^{k(n)} \alpha_{i}^{n} v_{n+i}, \quad\left(\alpha_{i}^{n} \geqq 0, \sum_{i=0}^{k(n)} \alpha_{i}^{n}=1\right),
$$

such that

$$
\iint_{Q}\left|w_{n}(\xi, \eta)-v_{0}(\xi, \eta)\right| d \xi d \eta \rightarrow 0 \quad \text { as } \quad n \rightarrow+\infty
$$

Thus, passing to a subsequence (without change of notation), we can assume that $w_{n}(x, y) \rightarrow v_{0}(x, y)$ for each $(x, y) \in Q \backslash Q_{0}$, where $\mu\left(Q_{0}\right)=0$. Let $\tilde{Q} \supset Q_{0}, \mu(\tilde{Q})=0$, be such that for every $(x, y) \in Q \backslash \widetilde{Q}$, the multifunction $F(x, y, \cdot)$ is u.s.c. and, moreover, $v_{n}(x, y) \in F\left(x, y, z_{n}(x, y)\right)$ for $n=1,2, \ldots$

Let $(x, y) \in Q \backslash \widetilde{Q}$ and let $\varepsilon>0$. Since $F\left(x, y\right.$,.) is u.s.c. at $z_{0}(x, y)$, there exists $n_{0}=$ $n_{0}(x, y, \varepsilon) \in \mathbb{N}$ such that $v_{n}(x, y) \in F\left(x, y, z_{0}(x, y)\right)+\varepsilon B$ for every $n \geqq n_{0}$. This implies $w_{n}(x, y) \in F\left(x, y, z_{0}(x, y)\right)+\varepsilon B$ for $n \geqq n_{0}$. From this we deduce that $v_{0}(x, y) \in F\left(x, y, z_{0}(x, y)\right)$. Since $(x, y)$ is arbitrary in $Q \backslash \tilde{Q}$ it is proved that $v_{0}(x, y) \in F\left(x, y, z_{0}(x, y)\right)$ for a.a. $(x, y) \in Q$. Moreover, from (4) for each $(x, y) \in Q$ we have

$$
\sum_{i=0}^{k(n)} \alpha_{i}^{n} z_{n+i}(x, y)=\phi(x)+\psi(y)-\phi(0)+\int_{0}^{x} \int_{0}^{y} w_{n}(\xi, \eta) d \xi d \eta
$$

and so, letting $n \rightarrow+\infty$, we get

$$
z_{0}(x, y)=\phi(x)+\psi(y)-\phi(0)+\int_{0}^{x} \int_{0}^{y} v_{0}(\xi, \eta) d \xi d \eta
$$

Hence $z_{0} \in \zeta_{F}$ and the compactness of $\zeta_{F}$ is established.

It remains to prove that $\zeta_{F}$ is contractible. Let $u_{0}$ be the (unique) solution of the Darboux problem $z_{x y}=g(x, y, z), z(x, 0)=\phi(x), z(0, y)=\psi(y)$. Let $u \in \zeta_{F}$ be arbitrary. For 
$t \in[0,1)$ consider the Darboux problem

$$
\begin{aligned}
z_{x y} & =g(x, y, z) \\
z(x, t) & =u(x, t), \quad z(t, y)=u(t, y), \quad(x, y) \in Q_{t}
\end{aligned}
$$

where $Q_{t}=[t, 1] \times[t, 1]$. Denote by $z^{(t)}: Q_{t} \rightarrow R^{d}$ the (unique) solution of problem (5).

For $t \in[0,1)$ define $u^{(t)}: Q \rightarrow R^{d}$ by

$$
u^{(t)}(x, y)=\left\{\begin{array}{lll}
z^{(t)}(x, y), & \text { if } & (x, y) \in Q_{t} \\
u(x, y), & \text { if } & (x, y) \in Q \backslash Q_{t} .
\end{array}\right.
$$

Moreover, set $u^{(1)}=u$. Observe that $u^{(0)}=u_{0}$.

We claim that for every $t \in[0,1], u^{(t)}$ is a solution of problem (3). Indeed, let $t \in[0,1]$. For every $(x, y) \in Q \backslash Q_{\mathrm{t}}$ we have

$$
u^{(t)}(x, y)=u(x, y)=\phi(x)+\psi(y)-\phi(0)+\int_{0}^{x} \int_{0}^{y} v(\xi, \eta) d \xi d \eta
$$

where $v(\xi, \eta) \in F\left(\xi, \eta, u^{(t)}(\xi, \eta)\right)$ for a.a. $(\xi, \eta) \in Q \backslash Q_{t}$.

For $(x, y) \in Q_{t}$ we have

$$
u^{(t)}(x, y)=z^{(t)}(x, y)=u(x, t)+u(t, y)-u(t, t)+\int_{t}^{x} \int_{t}^{y} v^{(t)}(\xi, \eta) d \xi d \eta
$$

where $v^{(t)}(\xi, \eta)=g\left(\xi, \eta, z^{(t)}(\xi, \eta)\right) \in F\left(\xi, \eta, z^{(t)}(\xi, \eta)\right)$ and so $v^{(t)}(\xi, \eta) \in F\left(\xi, \eta, u^{(t)}(\xi, \eta)\right)$ for a.a. $(\xi, \eta) \in Q_{r}$. From (7), by virtue of $(6)$, we have

$$
\begin{aligned}
u^{(t)}(x, y)= & \phi(x)+\psi(t)-\phi(0)+\int_{0}^{x} \int_{0}^{t} v(\xi, \eta) d \xi d \eta \\
& +\phi(t)+\psi(y)-\phi(0)+\int_{0}^{t} \int_{0}^{y} v(\xi, \eta) d \xi d \eta \\
& -\phi(t)-\psi(t)+\phi(0)-\int_{0}^{t} \int_{0}^{t} v(\xi, \eta) d \xi d \eta+\int_{t}^{x} \int_{t}^{y} v^{(t)}(\xi, \eta) d \xi d \eta \\
= & \phi(x)+\psi(y)-\phi(0)+\int_{0}^{x} \int_{0}^{y}\left[\chi_{Q \backslash Q_{t}}(\xi, \eta) v(\xi, \eta)+\chi_{Q_{t}}(\xi, \eta) v^{(t)}(\xi, \eta)\right] d \xi d \eta
\end{aligned}
$$

where $\chi_{A}$ denotes the characteristic function of $A$. It follows that $u^{(t)}$ is a solution of (3). Thus $u^{(t)} \in \zeta_{F}$ for each $u \in \zeta_{F}$ and $t \in I$.

Now define the function $h: I \times \zeta_{F} \rightarrow \zeta_{F}$ by $h(t, u)=u^{(t)}$. Suppose that $I \times \zeta_{F}$ is given the 
metric $\max \left\{\left|t_{1}-t_{2}\right|,\left\|u_{1}-u_{2}\right\|\right\}, \quad\left(t_{1}, u_{1}\right),\left(t_{2}, u_{2}\right) \in I \times \zeta_{F} \quad\left(\left\|u_{1}-u_{2}\right\|=\max _{(x, y) \in Q} \mid u_{1}(x, y)-\right.$ $\left.u_{2}(x, y) \mid\right)$. We are going to prove that $h$ is continuous.

Under our assumptions $\zeta_{F}$ is a bounded subset of $C\left(Q, R^{d}\right)$, thus there is a constant $m>0$ such that for every $u \in \zeta_{F}$ one has $u(x, y) \in m B,(x, y) \in Q$. Since the set $Q \times m B$ is compact and convex, the restriction of the function $g$ to $Q \times m B$ is lipschitzian with some constant $L>0$.

Let $(\tilde{t}, \tilde{u}) \in I \times \zeta_{F}$. Let $\varepsilon>0$ and choose $0<\delta<\varepsilon /\left(7 e^{L}\right)$. Let $\tau>0$ be so that $\iint_{\Delta} \alpha(\xi, \eta) d \xi d \eta<\delta$, where $\Delta=\{(x, y) \in Q \mid x, y \in[\tilde{t}-\tau, \tilde{t}+\tau]\}$. Let $(t, u) \in I \times \zeta_{F}$ be such that $|t-\tilde{t}|<\tau,\|u-\tilde{u}\|<\delta$. Let $t>\tilde{t}$ (the proof is similar when $t<\tilde{t}$ ).

Suppose $(x, y) \in Q_{i}$. As

$$
\begin{aligned}
& h(t, u)(x, y)=u(x, t)+u(t, y)-u(t, t)+\int_{t}^{x} \int_{t}^{y} g(\xi, \eta, h(t, u)(\xi, \eta)) d \xi d \eta \\
& h(\tilde{t} \tilde{u})(x, y)=\tilde{u}(x, \tilde{t})+\tilde{u}(\tilde{t}, y)-\tilde{u}(\tilde{t}, \tilde{t})+\int_{\tilde{t}}^{x} \int_{t}^{y} g(\xi, \eta, h(\tilde{t}, \tilde{u})(\xi, \eta)) d \xi d \eta
\end{aligned}
$$

we have

$$
\begin{aligned}
& |h(t, u)(x, y)-h(\tilde{t}, \tilde{u})(x, y)| \leqq|u(x, t)-\tilde{u}(x, \tilde{t})|+|u(t, y)-\tilde{u}(\tilde{t}, y)| \\
& \quad+|u(t, t)-\tilde{u}(\tilde{t}, \tilde{t})|+\iint_{\Delta} \alpha(\xi, \eta) d \xi d \eta \\
& \quad+\int_{t}^{x} \int_{t}^{y}|g(\xi, \eta, h(t, u)(\xi, \eta))-g(\xi, \eta, h(\tilde{t}, \tilde{u})(\xi, \eta))| d \xi d \eta \\
& \left.\quad<2 \delta+2 \delta+2 \delta+\delta+L \int_{t}^{x} \int_{t}^{y} \mid h(t, u)(\xi, \eta)-h(\tilde{t}, \tilde{u})(\xi, \eta)\right) \mid d \xi d \eta .
\end{aligned}
$$

From this, using Gronwall's inequality, we obtain $|h(t, u)(x, y)-h(\tilde{t}, \tilde{u})(x, y)| \leqq 7 \delta e^{L}<\varepsilon$.

Suppose $(x, y) \in Q_{\tilde{t}} \backslash Q_{t}$. As $h(\tilde{t}, \tilde{u})(x, y)$ is still given by (8) while

$$
h(t, u)(x, y)=u(x, y)=u(x, \tilde{t})+u(\tilde{t}, y)-u(\tilde{t}, \tilde{t})+\int_{\tilde{t}}^{x} \int_{\tilde{t}}^{y} g(\xi, \eta, u(\xi, \eta)) d \xi d \eta
$$

we have

$$
\begin{aligned}
& |h(t, u)(x, y)-h(\tilde{t}, \tilde{u})(x, y)| \leqq|u(x, \tilde{t})-\tilde{u}(x, \tilde{t})|+|u(\tilde{t}, y)-\tilde{u}(\tilde{t}, y)| \\
& \quad+|u(\tilde{t}, \tilde{t})-\tilde{u}(\tilde{t}, \tilde{t})|+2 \iint_{\Delta} \alpha(\xi, \eta) d \xi d \eta<\delta+\delta+\delta+2 \delta=5 \delta<\varepsilon
\end{aligned}
$$

Finally, if $(x, y) \in Q \backslash Q_{\tilde{t}}$ we have $|h(t, u)(x, y)-h(\tilde{t}, \tilde{u})(x, y)|=|u(x, y)-\tilde{u}(x, y)|<\delta<\varepsilon$. Hence $|h(t, u)(x, y)-h(\tilde{t}, \tilde{u})(x, y)|<\varepsilon$ for every $(x, y) \in Q$, which implies $\|h(t, u)-h(\tilde{t}, \tilde{u})\| \leqq \varepsilon$. This shows that $h$ is continuous at $(\tilde{t}, \tilde{u})$. As $(\tilde{t}, \tilde{u})$ is arbitrary, $h$ is continuous on $I \times \zeta_{F}$. Moreover, $h(0, u)=u_{0}$ and $h(1, u)=u$, for every $u \in \zeta_{F}$. Hence $\zeta_{F}$ is contractible and the proof of Lemma 4 is complete. 


\section{Proof of the Theorem}

By Lemma 1 , for every $k \in \mathbb{N}$ there is a locally lipschitzian function $g_{k}: Q \times R^{d} \rightarrow R^{d}$ such that

$$
\iint_{Q} \sup _{z \in R^{d}}\left|g_{k}(\xi, \eta, z)-f(\xi, \eta, z)\right| d \xi d \eta \leqq \frac{1}{2^{k}}
$$

For $n \in \mathbb{N}$ define $\tilde{\lambda}_{n}: Q \rightarrow[0,+\infty]$ by

$$
\tilde{\lambda}_{n}(x, y)=\sum_{k \geqq n} \sup _{z \in R^{d}}\left|g_{k}(x, y, z)-f(x, y, z)\right|
$$

Note that each $\tilde{\lambda}_{n}$ is integrable on $Q$. Consequently there is a null set $Q_{0} \subset Q$ such that $\tilde{\lambda}_{n}(x, y)$ is finite for every $(x, y) \in Q \backslash Q_{0}$, and every $n \in \mathbb{N}$. Define $\lambda_{n}: Q \rightarrow \mathbb{R}$ by

$$
\lambda_{n}(x, y)=\left\{\begin{array}{lll}
\tilde{\lambda}_{n}(x, y), & \text { if } & (x, y) \in Q \backslash Q_{0} \\
0, & \text { if } \quad(x, y) \in Q_{0}
\end{array}\right.
$$

For $n \in \mathbb{N}$ define the multifunction $G_{n}: Q \times R^{d} \rightarrow \mathscr{K}$ by

$$
G_{n}(x, y, z)=f(x, y, z)+\lambda_{n}(x, y) B
$$

Clearly $G_{n}$ satisfies hypotheses $\left(\mathrm{H}_{1}\right),\left(\mathrm{H}_{2}\right),\left(\mathrm{H}_{3}\right)$ (the latter with $\alpha(x, y)+\lambda_{n}(x, y)$ in the place of $\alpha(x, y))$. Moreover $g_{n}(x, y, z) \in G_{n}(x, y, z)$ for each $(x, y, z) \in\left(Q \backslash Q_{0}\right) \times R^{d}$.

Consider the problem

$$
\begin{gathered}
z_{x y} \in G_{n}(x, y, z) \\
z(x, 0)=\phi(x), \quad z(0, y)=\psi(y) .
\end{gathered}
$$

Let $\zeta_{G_{n}}$ denote the set of all solutions $z: Q \rightarrow R^{d}$ of problem (9). By virtue of Lemma 4 (with $g=g_{n}, F=G_{n}$ ) the set $\zeta_{G_{n}}$ is nonempty, compact and contractible. Clearly $\zeta_{G_{1}} \supset \zeta_{G_{2}} \supset \ldots$, for $G_{1}(x, y, z) \supset G_{2}(x, y, z) \supset \ldots$ for each $(x, y, z) \in Q \times R^{d}$. By Lemma 2 , $\widetilde{\zeta}=\bigcap_{n=1}^{\infty} \zeta_{G_{n}}$ is an $R_{\delta}$-set in $C\left(Q, R^{d}\right)$. To finish the proof it suffices to show that $\zeta_{f}=\zeta$.

It is obvious that $\zeta_{f} \subset \tilde{\zeta}$. To see the reverse inclusion suppose that $z \in \zeta$. Let $n \in \mathbb{N}$. For each $(x, y) \in Q$ we have

$$
z(x, y)=\phi(x)+\psi(y)-\phi(0)+\int_{0}^{x} \int_{0}^{y} v_{n}(\xi, \eta) d \xi d \eta
$$

where $v_{n}(\xi, \eta) \in G_{n}(\xi, \eta, z(\xi, \eta))$ for a.a. $(\xi, \eta) \in Q$. Hence $v_{n}(\xi, \eta)=f(\xi, \eta, z(\xi, \eta))+w_{n}(\xi, \eta)$, where $w_{n}$ is a measurable function satisfying $w_{n}(\xi, \eta) \in \lambda_{n}(\xi, \eta) B$ for a.a. $(\xi, \eta) \in Q$. 
Consequently we have

$$
\begin{gathered}
\left|z(x, y)-\phi(x)-\psi(y)+\phi(0)-\int_{0}^{x} \int_{0}^{y} f(\xi, \eta, z(\xi, \eta)) d \xi d \eta\right| \\
\leqq \int_{0}^{x} \int_{0}^{y} \lambda_{n}(\xi, \eta) d \xi d \eta \leqq \frac{1}{2^{n-1}} .
\end{gathered}
$$

Since $n \in \mathbb{N}$ is arbitrary, we conclude that $z \in \zeta_{f}$. This completes the proof.

\section{REFERENCES}

1. A. Aronszajn, Le correspondant topologique de l'unicité dans la théorie des équations différentielles, Ann. Math. 43 (1942), 730-738.

2. F. S. De Blasi and J. MyjaK, Orlicz type category results for differential equations in Banach spaces, Comm. Math. 23 (1983), 193-197.

3. N. Dunford and J. T. Schwartz, Linear operators, Part I (Interscience, New York and London, 1958).

4 L. Gónniewicz and T. Pruszko, On the set of solutions of the Darboux problem for some hyperbolic equations, Bull. Acad. Polon. Sci., Sér. Sci. Math. Astronom. Phys. 38 (1980), 279-285.

5. M. Hukuhara, Sur les systèmes des équations différentielles ordinaires, Jap. J. Math. 5 (1928), 345-350.

6. D. M. Hyman, On decreasing sequence of compact absolute retracts, Fund. Math. 64 (1969), 91-97.

Dipartimento di Matematica

UnIVERSITÁ dI ROMA II

VIA O. RAIMONDO

00173 ROMA

ITALY
Instytut Matematykı AGH

Al. Mickiewicza 30 30-059 KRAKOW

POLAND 